\title{
Vibrational overtone spectroscopy of cold trapped hydroxyl anions
}

\author{
Olga Lakhmanskaya, ${ }^{*}$ Malcolm Simpson $\odot$, and Roland Wester $\odot^{\dagger}$ \\ Institut für Ionenphysik und Angewandte Physik, Universität Innsbruck, Technikerstraße 25, 6020 Innsbruck, Austria
}

(Received 13 February 2020; accepted 8 June 2020; published 16 July 2020)

\begin{abstract}
The second vibrational overtone transition, $v^{\prime}, J^{\prime}=3,1 \leftarrow v, J=0,0$, in $\mathrm{OH}^{-}$confined in a 22-pole radio frequency ion trap has been measured using a laser induced reaction with molecular hydrogen. The resultant spectral line is found at $10150.94(2) \mathrm{cm}^{-1}$ and the excitation rate of this overtone excitation is obtained, which sensitively probes the molecular potential surface. The Doppler broadened line profile is used to determine the translational temperature of the ions in buffer gas temperatures ranging from 9(1) to 52(1) K. The translational temperatures closely follow those of the buffer gas over this range, including an offset caused by radio frequency heating. In comparison with previous measurements of the rotational temperatures of trapped ions, we show that translational and rotational temperatures do not equilibrate at the low buffer gas temperatures.
\end{abstract}

DOI: 10.1103/PhysRevA.102.012809

\section{INTRODUCTION}

Precision spectroscopy of trapped molecular ions is a burgeoning field with applications in tests of fundamental physical constants, quantum coherence studies, ultracold chemistry, and astrochemistry. Proposals for spectroscopy on the simplest diatomic ions $\mathrm{H}_{2}{ }^{+}$and $\mathrm{HD}^{+}$have suggested they may be applied to such problems as the electron dipole moment [1], the proton-to-electron mass ratio [2], fundamental tests of QED effects, and the search for a fifth force [3,4]. Using ions confined in radio frequency traps, extremely sensitive molecular spectroscopy can be performed. Vibrational transitions in homonuclear diatomics, which are dipole forbidden but allowed by electric quadrupole transitions, have been observed on $\mathrm{N}_{2}{ }^{+}$[5] and nondestructive quantum state detection has been achieved [6,7]. Buffer gas cooled ions confined in multipole ion traps [8-10] have opened up the field to a variety of complex molecular ions, which has seen many applications in astrochemistry. Notably, it was first shown that $\mathrm{C}_{60}{ }^{+}$is a carrier of four diffuse interstellar bands [11]. Our group has performed rotational spectroscopy on the triatomic anion $\mathrm{NH}_{2}{ }^{-}$[12] precluding the tentative assignment that this species supports an interstellar absorption feature. The sensitivity in ion-molecule studies within Coulomb crystals has also allowed for the testing of non-Langevin behavior at ultracold temperatures [13]. In proton-transfer between sympathetically cooled $\mathrm{N}_{2} \mathrm{H}^{+}$and rotationally cold $\mathrm{H}_{2} \mathrm{O}$, nuclear spin isomer-dependent reaction rates were reported [14].

Recently, the study of overtone transitions to higher lying vibrational levels has been drawing growing attention, be-

\footnotetext{
*Present address: Russian Quantum Center, Bolshoy Bulvar 30, bld. 1, Moscow 121205, Russia.

†roland.wester@uibk.ac.at

Published by the American Physical Society under the terms of the Creative Commons Attribution 4.0 International license. Further distribution of this work must maintain attribution to the author(s) and the published article's title, journal citation, and DOI.
}

cause this provides an avenue for precision tests of the protonto-electron mass ratio [15]. However, sensitive techniques are required as the absorption rates drop substantially, easily one or two orders of magnitude for each overtone. A focus is on the hydrogen molecular ion, which has been investigated by cotrapping it with laser-cooled $\mathrm{Be}^{+}$in a radio frequency ion trap [4]. A proposal for the spectroscopy on $\mathrm{TeH}^{+}$suggests that sensitivity of measurements for varying $\mu=m_{e} / m_{p}$ could be brought to the $1 \times 10^{-18} /$ year level [16]. Vibrational overtone spectroscopy has also been used to study the cations $\mathrm{H}_{3}{ }^{+}$and $\mathrm{D}_{2} \mathrm{H}^{+}[17,18]$. Specifically, measurements on $\mathrm{H}_{3}{ }^{+}$, which has a triangular equilibrium geometry, have been used to probe the doubly degenerate bending mode at the barrier to linearity [19]. To achieve highest spectral resolution, twophoton overtone spectroscopy is being pursued $[2,20]$.

In order to sensitively probe transitions in molecular ions, a number of action-spectroscopic methods have been developed. Rovibrational transitions have been investigated using the techniques of laser induced reactions (LIRs) [21], infrared multiphoton dissociation (IRMPD) [22], and messenger tagging [23] whereas purely rotational transitions have been investigated using laser induced inhibition of complex growth (LIICG) [24]. More recently a double resonance IR-THz LIR [21] scheme has been presented which may be applied to the full range of cationic and anionic species. Probing schemes involving photodetachment of the extra electron have also been put to use in the case of trapped molecular anions [12,25]. The application of a neutral, inert buffer gas to a trapped ion setup allows for the cooling of both the translational and internal degrees of freedom $[8,10]$. At low temperatures, this allows for full quantum state preparation of rotational levels. Combined with a photodetachment scheme this has been used to investigate rotational state changing collision processes in trapped $\mathrm{OH}^{-}$in a helium buffer gas [26].

Here we present vibrational overtone spectroscopy on the $\mathrm{OH}^{-}$molecular anion. Using a laser-induced reaction to probe the absorption, we have found the third harmonic of the $\mathrm{OH}$ stretch vibration and determined its frequency and absorption strength. This allows us to benchmark high-level quantum 


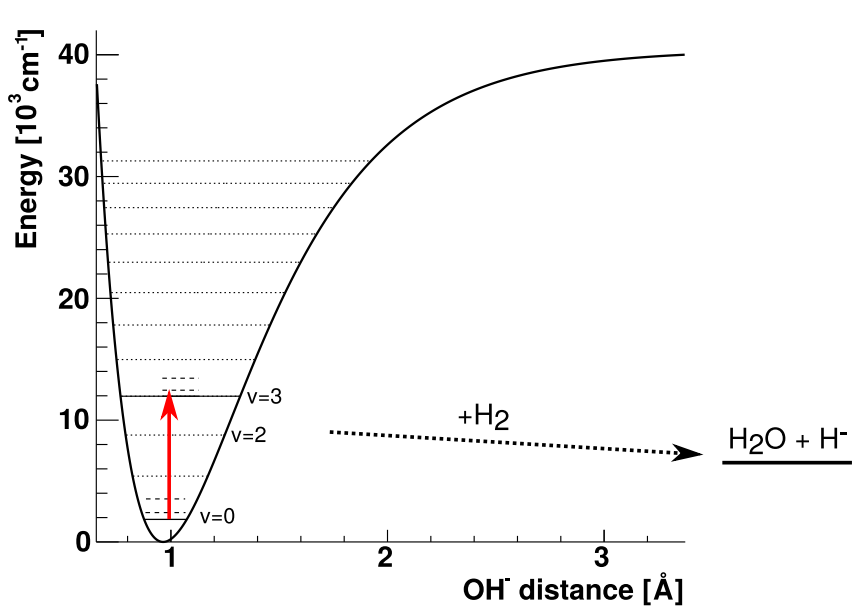

FIG. 1. Potential energy curve of $\mathrm{OH}^{-}$with vibrational and rotational energy levels (the rotational energy level separation is magnified). The red arrow shows the second vibrational overtone $v^{\prime}, J^{\prime}=3,1 \leftarrow v, J=0,0$ probed in this work.

chemical calculations of the anharmonicity of the $\mathrm{OH}^{-}$bond, including relativistic corrections to the interaction potential. The simple rotational structure in its ${ }^{1} \Sigma^{+}$ground state makes $\mathrm{OH}^{-}$an interesting candidate for precision spectroscopy, unperturbed by hyperfine splittings. The $s$-wave threshold detachment of $\mathrm{OH}^{-}$has been extensively studied through photodetachment $[27,28]$ and deviations from the Wigner threshold law have been observed due to the electron-dipole interaction [29,30]. With its large rotational constant, $\mathrm{OH}^{-}$ has also been used to probe the rotational thermalization by buffer gas cooling [26,31] and in cryogenic storage rings $[32,33]$. In buffer gas at low temperatures, significant deviations of the rotational temperature from the thermal bath have been seen [34]. In the present work we are able to resolve the Doppler profile at different buffer gas temperatures in the ion trap. This has allowed us to determine the ion translational temperature that can be compared with the previously measured rotational temperatures. We also compare the measured temperatures to numerical simulations. This enables an improved understanding of the buffer gas thermalization process in radio frequency multipole ion traps.

\section{METHODS}

The $\mathrm{OH}^{-}$fundamental vibrational mode has previously been measured [21] using the LIR method. We use the same reaction to probe the overtone transition,

$$
\mathrm{OH}^{-}(v>1)+\mathrm{H}_{2}+h v \rightarrow \mathrm{H}_{2} \mathrm{O}+\mathrm{H}^{-},
$$

by driving the rovibrational overtone transition $v^{\prime}, J^{\prime}=$ $3,1 \leftarrow v, J=0,0$ (see Fig. 1). To estimate the expected transition frequency, we use the theoretically calculated spectroscopic constants that were derived from a potential curve that included quadrupole excitations and scalar relativistic effects [35]. The transition frequencies from this high-level calculation show excellent agreement for the fundamental vibrational transitions that have previously been measured [21,36] (see Table I). The unobserved 3, $1 \leftarrow 0,0$ transition is predicted to lie at $10149.5 \mathrm{~cm}^{-1}$.

The experimental setup was operated similarly to our previous work [25]: $\mathrm{OH}^{-}$ions are produced in a pulsed plasma discharge in water vapor carried in a He jet. The ions are extracted perpendicularly by applying a short voltage pulse to a set of Wiley-McLaren electrodes. This allows us to separate ionized species by mass in terms of time of flight and to load only specific masses into the 22-pole ion trap [37]. Multiple ion pulses are loaded within a few hundred milliseconds, which improves the stability of the trapped ion signal. The experiment was performed at different trap temperatures ranging from 9(1) to 52(1) K. At these temperatures the ions were cooled via collisions with $n-\mathrm{H}_{2}$ buffer gas. The constant $\mathrm{H}_{2}$ density of between 1 and $4 \times 10^{12} \mathrm{~cm}^{-3}$ guarantees thermalization on millisecond timescales. After storage, the ions are extracted, mass selected, and then detected on a microchannel plate detector. To record vibrational spectra, the described experimental cycle is repeated for different laser frequencies.

The radiation was provided by a commercially available Ti:sapphire laser system (Matisse DR, Sirah). The laser power is in the range $300-400 \mathrm{~mW}$ at the wavelength of $10150 \mathrm{~cm}^{-1}$. The laser beam propagates along the trap axis and enters through the end-cap electrodes. To align the laser with the trap axis we used separate photodetachment measurements of $\mathrm{O}^{-}$anions at $760 \mathrm{~nm}$. The wavelength was detected via a HighFinesse WS7 wave meter, which has an absolute accuracy of $2 \times 10^{-3} \mathrm{~cm}^{-1}$. While being trapped for about $2-3 \mathrm{~s}$ the ions were exposed to the laser radiation. Reaction (1) is endothermic and requires about $3200 \mathrm{~cm}^{-1}$ of energy to take place [38-41]. The vibrational energy gained by the overtone excitation is therefore more than sufficient to drive the reaction. Furthermore, the reaction rate with $\mathrm{H}_{2}$ is much faster than the radiative lifetime of the excited vibrational levels, which provides essentially unit detection efficiency for every overtone transition.

\section{RESULTS AND DISCUSSION}

\section{A. Vibrational overtone transitions}

To find the overtone transition, the excitation laser was scanned over a broad frequency range. For this search the ion trapping and exposure time was set to $100 \mathrm{~s}$ to minimize the influence of ion number fluctuations in the trap. The overtone excitation was detected about $1.4 \mathrm{~cm}^{-1}$

TABLE I. Rovibrational line positions for the fundamental and second overtone vibrational transitions from this and previous experimental and theoretical studies.

\begin{tabular}{|c|c|c|c|c|c|}
\hline$\left(v^{\prime}, J^{\prime}\right) \leftarrow(v, J)$ & This work & Calculated [35] & Observed [36] & Estimated [36] & Observed [21] \\
\hline$(1,1) \leftarrow(0,0)$ & & 3591.57 & $3591.5254(20)$ & & $3591.5265(10)$ \\
\hline$(3,1) \leftarrow(0,0)$ & $10150.942(2)(22)$ & 10149.5 & & 10151.11 & \\
\hline
\end{tabular}


$(43 \mathrm{GHz})$ away from the calculated line position. Once found, high-resolution scans with 2-3 s trapping time were performed for different trap temperatures, which are shown in Fig. 2. The transition frequencies and widths were identified using the fit function,

$$
I(v)=\left(p_{1} v+p_{0}\right) \times e^{-p_{2} \times \exp \left(-\frac{m c^{2}}{2 k T}\left[\frac{v-v_{0}}{v_{0}}\right]^{2}\right) t},
$$

which describes a Doppler-broadened (Gaussian) line profile modified by the decay rate of the ions in the trap superimposed on a first-order polynomial to account for any overall signal drifts during the measurement. Given the thermalization in the buffer gas, we use a Maxwell-Boltzmann distribution for the

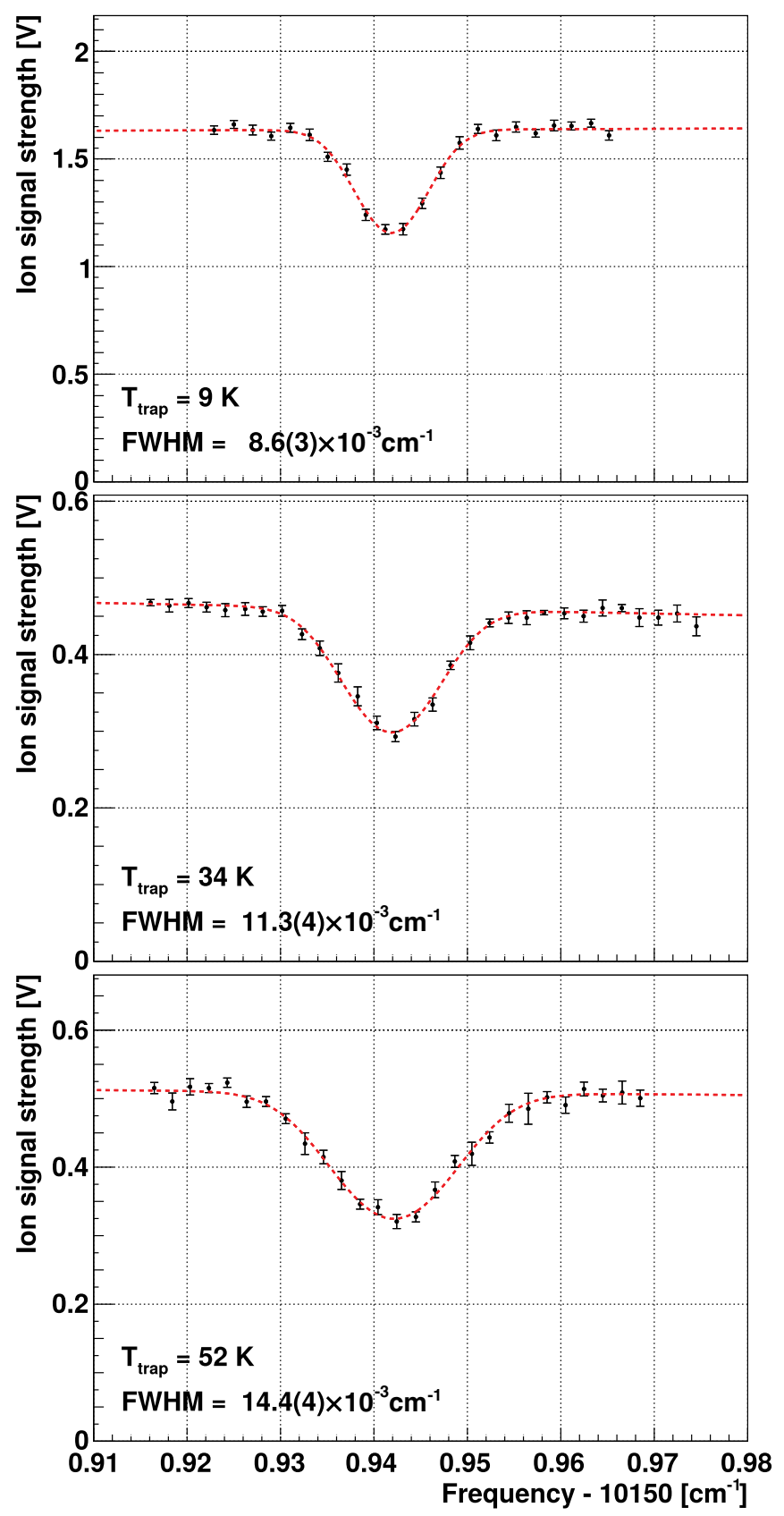

FIG. 2. LIR line of the overtone transition of $\mathrm{OH}^{-} v^{\prime}$, $J^{\prime}=3,1 \leftarrow v, J=0,0$. The line was measured at the trap temperatures (from the top to the bottom) of 9,34 , and $52 \mathrm{~K}$. ion velocities at any time of the experiment and a Gaussian line profile.

As a result, we determine the second overtone transition frequency of $\mathrm{OH}^{-} v^{\prime}, J^{\prime}=3,1 \leftarrow v, J=0,0$, as the average over a total of 21 individual measurements, to be $10150.942(2)(20) \mathrm{cm}^{-1}$ (see Table I). The first error value indicates the statistical error of the fit and the second represents the systematic accuracy limited by the absolute accuracy of our wave meter.

This transition frequency agrees almost perfectly with the prediction that was calculated by Martin [35]. The small difference of $1.4 \mathrm{~cm}^{-1}$, or $10^{-4}$ relative deviation, shows the quality of the calculation, which included high level calculations of the potential energy curve and the most important relativistic correction. To explain the remaining difference, new advanced quantum chemical calculations will be required, which may have to include higher order relativistic or nonadiabatic corrections. This shows the potential for high resolution overtone spectroscopy as benchmark for molecular structure calculations.

\section{B. Excitation rates}

To extract the overtone transition strength, we have measured the ion loss rates following overtone excitation. In steady state the $\mathrm{OH}^{-}$losses proceed with the excitation rate of the overtone transition $k_{03}$, given that the excited ions react with $\mathrm{H}_{2}$ with a much faster rate than the excitation rate. The time evolution of the ion signal with and without the resonant excitation laser present is shown in Fig. 3. For data at $39 \mathrm{~K}$ trap temperature, we obtain a measured loss rate on resonance of $0.25(1) \mathrm{s}^{-1}$.

From the measured loss rate, we can extract the transition dipole moment $\mu$ or the Einstein $B$ factor for this overtone excitation. The excitation rate $k_{03}$ is calculated by integrating over the product of the Einstein $B_{03}$ factor, the spectral energy density of the laser beam overlap, and the spatial density distribution of the trapped ions in the addressed rotational

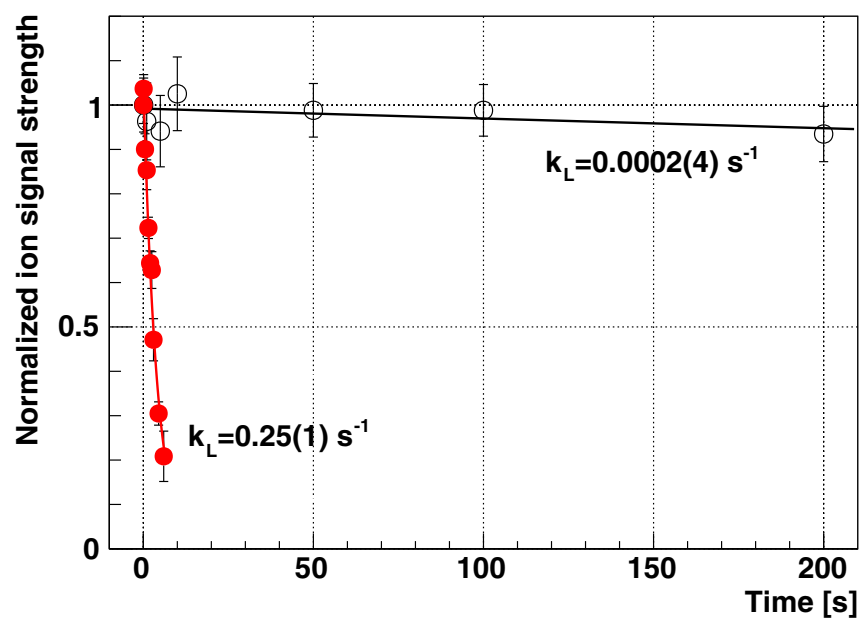

FIG. 3. $\mathrm{OH}^{-}$signal as a function of storage time. Black circles: Off-resonant laser interaction leading to very long storage time. Red circles: Resonant laser interaction driving the reaction with neutral $\mathrm{H}_{2}$. 
quantum state (see also Ref. [19]). The integral has to be taken over the volume of the ion-laser interaction and the frequency range of the transition. In our case the laser beam diameter and its spectral width are much smaller than the ion cloud diameter and the Doppler width of the excited transition, respectively. Thus the integral can be well approximated by the product

$$
k_{03}=p_{J=0} B_{03} \rho_{\text {avg }},
$$

where $p_{J=0}$ is the rotational ground state population, expected to be about 50\% at this temperature [34]. The spectral energy density $\rho_{\text {avg }}$ is calculated from the laser power averaged over the ion cloud diameter and the Doppler width of the transition, respectively. For a laser power of $0.3 \mathrm{~W}$, a cloud diameter of $8.5 \mathrm{~mm}$, and a measured Doppler width of $330 \mathrm{MHz}$ (see next section), a spectral energy density of $5 \times 10^{-14} \mathrm{~J} /\left(\mathrm{m}^{3} \mathrm{~Hz}\right)$ is obtained. The resulting Einstein $B_{03}$ factor is directly converted to the transition dipole moment, for which we obtain an estimate of $\mu_{03}=0.4 \times 10^{-3} \mathrm{D}$ (debye). This value characterizes the studied rovibrational transition. Often the rotationless transition dipole moment, which is corrected for the Hönl London factor, is reported. It amounts to $0.7 \times 10^{-3} \mathrm{D}$.

The measured transition dipole moment can be compared to calculated values for the first and second vibrational level, which amount to $0.0989 \mathrm{D}$ and $1.28 \times 10^{-3} \mathrm{D}$ for $\Delta v=1$ and 2 , respectively [42]. This shows that for the third vibrational level the transition dipole decreases only marginally compared to the large decrease from the fundamental to the second level. One finds that the dipole moment drops by a factor of about 80 from the first to the second vibrational level and only by a factor of about 2 from the second to the third vibrational level. We qualitatively rationalize this with the unusual shape of the dipole moment function of $\mathrm{OH}^{-}$, which decreases with increasing internuclear distance from the equilibrium value of $1.7 \AA$ and eventually changes sign near $1.4 \AA$ [42]. Together with the anharmonicity of the molecular potential curve this is found to render the second overtone transition similarly strong as the first overtone transition.

\section{Ion translational temperatures}

The measured line profiles agree well with the fit based on a Maxwell-Boltzmann velocity distribution. From its widths the ions' translational temperatures are obtained for different trap (and buffer gas) temperatures between 9 and $52 \mathrm{~K}$ (see Fig. 4). The translational ion temperatures follow those of the buffer gas, but lie always above the gas temperature. A fit to the data shows that, overall, they can be well described by a first-order polynomial with a linear coefficient of 1 and an additive offset of about $6 \mathrm{~K}$. At $9 \mathrm{~K}$ a significantly higher temperature of $20-25 \mathrm{~K}$ is observed.

Larger translational temperatures than that of the buffer gas have been known to occur in multipole traps for some time. For $\mathrm{OH}^{-}$in $\mathrm{H}_{2}$ a translational temperature of $29 \mathrm{~K}$ was measured at a trap temperature of $16 \mathrm{~K}$ [21]. Furthermore, a translational temperature of $10-20 \mathrm{~K}$ was identified for $\mathrm{NH}_{2} \mathrm{D}_{2}{ }^{+}$and $\mathrm{CH}^{+}$in a $4 \mathrm{~K}$ trap $[43,44]$. Our results show that this increase is well described by an offset, which is in agreement with numerical simulations of collision-induced radio frequency heating in radio frequency multipole ion

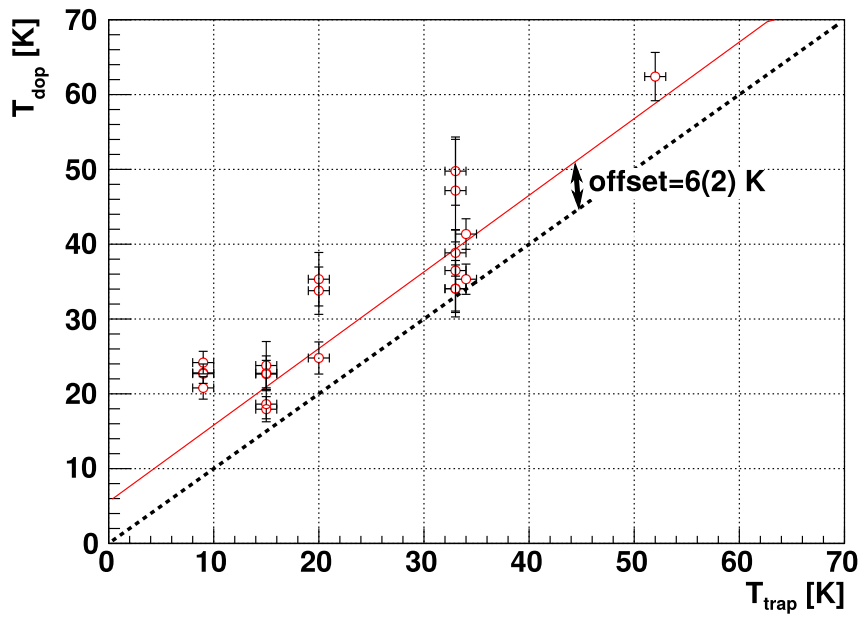

FIG. 4. The translational temperature of the $\mathrm{OH}^{-}$ions $T_{\text {Dop }}$, obtained from the Doppler fit, is plotted as a function of the trap (buffer gas) temperature $T_{\text {trap }}$.

traps, when nonideal effective potentials, for example due to the axially confining endcap electrodes, are included [45]. At the lowest buffer gas temperatures, additional radio frequency heating seems to occur, most likely because of ion trapping near patch potentials.

The measured translational ion temperatures are similar to the $\mathrm{OH}^{-}$rotational temperatures, which we recently measured in the same ion trap [34]. There we also found larger rotational temperatures than the temperature of the trap and the buffer gas. A larger rotational temperature of about $9 \mathrm{~K}$ was also observed for $\mathrm{CH}_{3}{ }^{+}$at $4 \mathrm{~K}$ trap temperature [46]. These results are in striking disagreement with the notion that the collision dynamics in the center-of-mass frame of the ions can be described by a properly averaged effective temperature

$$
T_{\text {eff }}=\frac{m_{\text {ion }} T_{\text {buffer }}+m_{\text {buffer }} T_{\text {ion }}}{m_{\text {ion }}+m_{\text {buffer }}},
$$

where $m_{\text {ion }}, m_{\text {buffer }}$ indicate the ion and buffer gas masses and $T_{\text {ion }}, T_{\text {buffer }}$ are the associated translational temperatures. Using this equation and our present results for the translational temperature, one obtains that the collisional ion temperature does not exceed $12 \mathrm{~K}$ at a trap (buffer gas) temperature of $10 \mathrm{~K}$. This is much smaller than the measured rotational ion temperature of about $25 \mathrm{~K}$ [34] and shows that a yet unexplained heating mechanism acts on the rotational degrees of freedom of the trapped ions that does not affect the translational temperature.

\section{CONCLUSION}

In conclusion, the second vibrational overtone of $\mathrm{OH}^{-}$ has been measured using a sensitive overtone excitation and laser-induced reaction scheme in a cryogenic ion trap. Only a very small difference of $1.4 \mathrm{~cm}^{-1}$ was found compared to the prediction from a high level $a b$ initio calculation [35]. Thus, the measurement serves as a benchmark for precise molecular structure calculations. We have also extracted the transition dipole moment for the second overtone excitation and found it to be unexpectedly large compared to the fundamental or 
first overtone transition. This is rationalized with the interplay of the dipole moment function and the anharmonicity of the molecular vibration.

By inspecting the Doppler widths at different trap temperatures, we analyzed the ion thermalization in the buffer gas. Overall, the translational ion temperatures were observed to follow the trap temperature, taking into account an additive offset of about $6 \mathrm{~K}$, in good agreement with expectations from collisional radio frequency heating. Only at the lowest buffer gas temperature a significantly larger radio frequency heating was found. The precise knowledge of the infrared overtone transitions and the simple rotational level scheme in the ${ }^{1} \Sigma^{+}$electronic ground state of $\mathrm{OH}^{-}$make this system an interesting candidate for two-photon precision spectroscopy. This may allow for unprecedented sub-Doppler resolution in molecular ion spectroscopy.

\section{ACKNOWLEDGMENT}

We thank Eric Endres for valuable discussions. This work has been supported by the Austrian Science Fund (FWF) through the Doctoral Programme Atoms, Light, and Molecules, Project No. W1259-N27, and through Project No. P29558-N36.
[1] W. B. Cairncross, D. N. Gresh, M. Grau, K. C. Cossel, T. S. Roussy, Y. Ni, Y. Zhou, J. Ye, and E. A. Cornell, Phys. Rev. Lett. 119, 153001 (2017).

[2] J.-P. Karr, L. Hilico, J. C. J. Koelemeij, and V. I. Korobov, Phys. Rev. A 94, 050501(R) (2016).

[3] S. Schiller, D. Bakalov, and V. I. Korobov, Phys. Rev. Lett. 113, 023004 (2014).

[4] J. Biesheuvel, J.-P. Karr, L. Hilico, K. S. E. Eikema, W. Ubachs, and J. C. J. Koelemeij, Nat. Commun. 7, 10385 (2016).

[5] M. Germann, X. Tong, and S. Willitsch, Nat. Phys. 10, 820 (2014).

[6] F. Wolf, Y. Wan, J. C. Heip, F. Gebert, C. Shi, and P. O. Schmidt, Nature (London) 530, 457 (2016).

[7] C. Chou, C. Kurz, D. B. Hume, P. N. Plessow, D. R. Leibrandt, and D. Leibfried, Nature (London) 545, 203 (2017).

[8] D. Gerlich, Phys. Scr. T59, 256 (1995).

[9] O. Asvany, J. Krieg, and S. Schlemmer, Rev. Sci. Instrum. 83, 093110 (2012).

[10] R. Wester, J. Phys. B 42, 154001 (2009).

[11] E. K. Campbell, M. Holz, D. Gerlich, and J. P. Maier, Nature (London) 523, 322 (2015).

[12] O. Lakhmanskaya, M. Simpson, S. Murauer, M. Nötzold, E. Endres, V. Kokoouline, and R. Wester, Phys. Rev. Lett. 120, 253003 (2018).

[13] B. R. Heazlewood, Mol. Phys. 117, 1934 (2019).

[14] A. Kilaj, H. Gao, D. Rösch, U. Rivero, J. Küpper, and S. Willitsch, Nat. Commun. 9, 2096 (2018).

[15] M. S. Safronova, D. Budker, D. DeMille, D. F. Jackson Kimball, A. Derevianko, and C. W. Clark, Rev. Mod. Phys. 90, 025008 (2018).

[16] M. G. Kokish, P. R. Stollenwerk, M. Kajita, and B. C. Odom, Phys. Rev. A 98, 052513 (2018).

[17] J. Mikosch, H. Kreckel, R. Wester, R. Plasil, J. Glosik, D. Gerlich, D. Schwalm, and A. Wolf, J. Chem. Phys. 121, 11030 (2004).

[18] J. Glosík, P. Hlavenka, R. Plašil, F. Windisch, D. Gerlich, A. Wolf, and H. Kreckel, Philos. Trans. R. Soc. A 364, 2931 (2006).

[19] H. Kreckel, D. Bing, S. Reinhardt, A. Petrignani, M. Berg, and A. Wolf, J. Chem. Phys. 129, 164312 (2008).

[20] R. Carollo, A. Frenett, and D. Hanneke, Atoms 7, 1 (2018).

[21] P. Jusko, O. Asvany, A.-C. Wallerstein, S. Brünken, and S. Schlemmer, Phys. Rev. Lett. 112, 253005 (2014).
[22] K. R. Asmis, N. L. Pivonka, G. Santambrogio, M. Brümmer, C. Kaposta, D. M. Neumark, and L. Wöste, Science 299, 1375 (2003).

[23] M. Brümmer, C. Kaposta, G. Santambrogio, and K. R. Asmis, J. Chem. Phys. 119, 12700 (2003).

[24] S. Chakrabarty, M. Holz, E. K. Campbell, A. Banerjee, D. Gerlich, and J. P. Maier, J. Phys. Chem. Lett. 4, 4051 (2013).

[25] S. Lee, D. Hauser, O. Lakhmanskaya, S. Spieler, E. S. Endres, K. Geistlinger, S. S. Kumar, and R. Wester, Phys. Rev. A 93, 032513 (2016).

[26] D. Hauser, S. Lee, F. Carelli, S. Spieler, O. Lakhmanskaya, E. S. Endres, S. S. Kumar, F. Gianturco, and R. Wester, Nat. Phys. 11, 467 (2015).

[27] P. A. Schulz, R. Mead, P. Jones, and W. Lineberger, J. Chem. Phys. 77, 1153 (1982).

[28] F. Goldfarb, C. Drag, W. Chaibi, S. Kröger, C. Blondel, and C. Delsart, J. Chem. Phys. 122, 014308 (2005).

[29] P. C. Engelking and D. R. Herrick, Phys. Rev. A 29, 2425 (1984).

[30] J. R. Smith, J. B. Kim, and W. C. Lineberger, Phys. Rev. A 55, 2036 (1997).

[31] R. Otto, A. von Zastrow, T. Best, and R. Wester, Phys. Chem. Chem. Phys. 15, 612 (2013).

[32] C. Meyer, A. Becker, K. Blaum, C. Breitenfeldt, S. George, J. Göck, M. Grieser, F. Grussie, E. A. Guerin, R. von Hahn, P. Herwig, C. Krantz, H. Kreckel, J. Lion, S. Lohmann, P. M. Mishra, O. Novotný, A. P. O’Connor, R. Repnow, S. Saurabh et al., Phys. Rev. Lett. 119, 023202 (2017).

[33] G. Eklund, K. Chartkunchand, E. K. Anderson, M. Kamińska, N. de Ruette, R. D. Thomas, M. K. Kristiansson, M. Gatchell, P. Reinhed, S. Rosén, A. Simonsson, A. Källberg, P. Löfgren, S. Mannervik, H. Zettergren, H. Cederquist, and H. T. Schmidt, J. Phys.: Conf. Ser. 875, 012016 (2017).

[34] E. S. Endres, G. Egger, S. Lee, O. Lakhmanskaya, M. Simpson, and R. Wester, J. Mol. Spectrosc. 332, 134 (2017).

[35] J. M. L. Martin, Spectrochim. Acta, Part A 57, 875 (2001).

[36] N. H. Rosenbaum, J. C. Owrutsky, L. M. Tack, and R. J. Saykally, J. Chem. Phys. 84, 5308 (1986).

[37] T. Best, R. Otto, S. Trippel, P. Hlavenka, A. von Zastrow, S. Eisenbach, S. Jezouin, R. Wester, E. Vigren, M. Hamberg, and W. D. Geppert, Astrophys. J. 742, 63 (2011).

[38] E. de Beer, E. H. Kim, D. M. Neumark, R. F. Gunion, and W. C. Lineberger, J. Phys. Chem. 99, 13627 (1995). 
[39] T. M. Miller, A. A. Viggiano, A. E. S. Miller, R. A. Morris, M. Henchman, J. F. Paulson, and J. M. Van Doren, J. Chem. Phys. 100, 5706 (1994).

[40] D. H. Zhang, M. Yang, M. A. Collins, and S.-Y. Lee, Proc. Natl. Acad. Sci. USA 99, 11579 (2002).

[41] D. Wang, J. Z. H. Zhang, and C. Hui Yu, Chem. Phys. Lett. 273, 171 (1997).

[42] H.-J. Werner, P. Rosmus, and E.-A. Reinsch, J. Chem. Phys. 79, 905 (1983).
[43] J. L. Doménech, P. Jusko, S. Schlemmer, and O. Asvany, Astrophys. J. 857, 61 (2018).

[44] J. L. Doménech, S. Schlemmer, and O. Asvany, Astrophys. J. 866, 158 (2018).

[45] D. Hauser, Photodetachment Studies of Molecular Anions in Multipole Ion Traps, Ph.D. thesis, University of Innsbruck, 2015, http://permalink.obvsg.at/UIB/AC11359682.

[46] O. Asvany, S. Thorwirth, B. Redlich, and S. Schlemmer, J. Mol. Spectrosc. 347, 1 (2018). 